\title{
Level spacing statistics of bidimensional Fermi liquids: II. Landau fixed point and quantum chaos
}

\author{
R. Mélin \\ CRTBT-CNRS, 38042 Grenoble BP 166X cédex France
}

\begin{abstract}
We investigate the presence of quantum chaos in the spectrum of the bidimensional Fermi liquid by means of analytical and numerical methods. This model is integrable in a certain limit by bosonization of the Fermi surface. We study the effect on the level statisticsof the momentum cutoff $\Lambda$ present in the bidimensional bosonization procedure. We first analyse the level spacing statistics in the $\Lambda$-restricted Hilbert space in one dimension. With $g_{2}$ and $g_{4}$ interactions, the level statistics are found to be Poissonian at low energies, and G.O.E. at higher energies, for a given cut-off $\Lambda$. In order to study this cross-over, a finite temperature is introduced as a way of focussing, for a large inverse temperature $\beta$, on the low energy many-body states, and driving the statistics from G.O.E. to Poissonian. As far as two dimensions are concerned, we diagonalize the Fermi liquid Hamiltonian with a small number of orbitals. The level spacing statistics are found to be Poissonian in the $\Lambda$-restricted Hilbert space, provided the diagonal elements are of the same order of magnitude as the off-diagonal matrix elements of the Hamiltonian.
\end{abstract}

To be published in Jour. Phys I (France) 


\section{Contents}

\begin{tabular}{llr}
\hline 1 & Introduction & 1
\end{tabular}

2 Level spacing statistics of 1 D spinless fermion systems in the $\Lambda$-restricted \begin{tabular}{|l|l|}
\hline Hilbert space & 5
\end{tabular}

2.1 Bosonization and level spacing statistics in the unrestricted Hilbert space (Luttinger liquid

$2.2 \quad$ Fourier transform of the gas spectrum and temperature-dependent level spacing statistics . . . . . . . . . . . . . . . . . . . . . 7

2.3 Level spacing statistics with $g_{2}$ interactions in the $\Lambda$-restricted Hilbert space $\quad 8$

2.4 Level spacing statistics with $g_{4}$ interactions in the $\Lambda$-restricted Hilbert space . 9

2.5 Cross-over scales . . . . . . . . . . . . . . . . . . . . . 10

3 Level spacing statistics of a 2 D spinless fermion system in the $\Lambda$-restricted \begin{tabular}{|ll}
\hline Hilbert space & 10
\end{tabular}

3.1 Bosonization of the bidimensional Fermi surface . . . . . . . . . . . . . . . . . 10

3.2 Level spacing statistics of a 2 D spinless fermion system in the $\Lambda$-restricted Hilbert space . . . . . . . . . . . . . . . . . . . 12

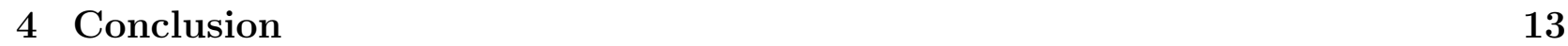

\section{Introduction}

The ideas of quantum chaos have recently been applied to the field of strongly correlated electron theory [1] [2] [3]. These methods allow a non perturbative description of the statistical properties of a Hamiltonian of strongly correlated electrons, and may be a tool to extract some information from finite size systems. The aim of this article is to understand whether the methods of quantum chaos can shed some new light on the problems of strongly correlated bidimensional Fermi systems. The question whether the bidimensional Hubbard model is a Fermi liquid or not is controversial. From a theoretical point of view, Anderson [母] suggests that the ground state of the bidimensional Hubbard model is similar to the one of the onedimensional Luttinger liquid, with $Z=0$ in the thermodynamic limit, whereas Engelbrecht and Randeria [5] argue that the Fermi-liquid theory is not violated, so that this question is controversial [6]. Numerical computations lead also to controversial answers. For instance, Dagotto et al [7] [8] observe quasiparticules in the bidimensional t-J model, whereas Sorella [9] emphasizes the Luttinger liquid behaviour. From the point of view of quantum chaos, one has to answer the question: do level spacing statistics contain information about the Fermi 
liquid behaviour or non Fermi liquid behaviour of the t-J model? Before analysing the level spacing statistics of the t-J model [10], we analyze in this paper models with a well established physical content, that is the Fermi liquid. As we shall see, we can answer only partially the previous question: one can detect integrable modes at low energy, but quantum chaos by itself does not give information as to whether these modes are quasiparticule modes or Luttinger liquid modes. However, there is one case in which one can conclude from quantum chaos: in the absence of integrable degrees of freedom at low energy, one can conclude to the absence of a Fermi liquid at low energy. Notice here the difference between the approach of [1] and our point of view. In [1], all the energy levels of the t-J model are analyzed on an equal footing, whereas we shall focus essentially on the low energy degrees of freedom of the Fermi liquid.

We have established in the first article of this series [11], that the level spacing statistics of the bidimensional Landau Hamiltonian are poissonian. In this model, the quasiparticules are in a one to one correspondence with the non interacting gas of spinless electron excitations, and thus occupy orbitals labelled by the same quantum numbers $k$ as in the case of the gas. The quasiparticules interact among themselves in a diagonal manner

$$
H\left[\left\{\delta n_{\mathbf{k}}\right\}\right]=\sum_{\mathbf{k}} \epsilon_{\mathbf{k}} \delta n_{\mathbf{k}}+\frac{1}{L^{D}} \sum_{\left\langle\mathbf{k}, \mathbf{k}^{\prime}\right\rangle} f_{\mathbf{k k}^{\prime}} \delta n_{\mathbf{k}} \delta n_{\mathbf{k}^{\prime}} .
$$

A numerical computation proved that the level spacing statistics of the Hamiltonian (1) are poissonian in two dimensions, and close to Poisson in one dimension, with our truncation of the Hilbert space. The notion of level spacing statistics seems to be relevant for quantum fluids, and one has to distinguish between one and two dimensions. The link between the breakdown of the Fermi liquid picture in one dimension and the level spacing statistics has already been studied in reference [13. As far as two dimensions are concerned, we showed in the paper I [11, that the Landau liquid was characterized by its generical integrability, namely by Poisson level spacing statistics in two dimensions. Again, the level spacing statistics are a good tool to see whether the Fermi system is at the Landau fixed point or not. For the liquid to be a Fermi liquid, one should be able to generate quasiparticules of the interacting system by a switching on procedure. In the framework of the Landau theory, the success of the switchingon procedure [14] [15] suggests the conservation of the number of conserved quantities at low energy during the switching-on procedure, namely that the level spacing statistics of the gas and of the interacting liquid belong to the same universality class. Typically, adding a non diagonal perturbation to (11) and obtaining a Gaussian Orthogonal Ensemble (G.O.E.) level spacing statistics would mean a departure from the Landau fixed point. To be at the Landau fixed point, one must exclude strong correlations between levels. The corresponding generic level spacing statistics are poissonian. However, the case of the one dimensional Landau Hamiltonian shows that the statistics may not be exactly poissonian, but close to a Poisson 
law. Poisson or close to Poisson level spacing statistics are not a sufficient condition for the system to exhibit quasiparticules since one could imagine a situation in which the same mechanism as in one dimension for the breakdown of the Fermi liquid holds, so that the level spacing statistics would remain poissonian even in the non Fermi liquid case. However, the adiabatic procedure, as emphasized by Anderson [14, is performed within a finite characteristic time $1 / \epsilon$, and the thermodynamic limit is taken for a finite $\epsilon$ which is then much larger than the typical level spacing. So the adiabatic continuation does not generate true eigenstates (for this to be the case, one should take the limit $\epsilon \rightarrow 0$ first, before the thermodynamic limit). This is why the notion of adiabatic continuation advocated to introduce Fermi liquid is weaker than a similar requirement for all the eigenstates taken separately. So, this is why we should ask whether a Fermi liquid follows Poisson or G.O.E. statistics.

The aim of the present paper is to study the level spacing statistics of the Hamiltonian of spinless electrons

$$
\begin{aligned}
H & =H^{0}+H^{1} \\
H^{0} & =\sum_{\mathbf{k}} \epsilon(\mathbf{k}) c_{\mathbf{k}}^{+} c_{\mathbf{k}} \\
H^{1} & =\frac{1}{2 V} \sum_{\mathbf{k}, \mathbf{k}^{\prime}, \mathbf{q}} f_{\mathbf{k}, \mathbf{k}^{\prime}, \mathbf{q}} c_{\mathbf{k}+\mathbf{q}}^{+} c_{\mathbf{k}^{\prime}-\mathbf{q}}^{+} c_{\mathbf{k}^{\prime}} c_{\mathbf{k}},
\end{aligned}
$$

where $V$ is the volume of the system. The Hamiltonian (1) describes a Fermi liquid for times inferior as the decay time of the quasiparticules, and leads to good thermodynamical predictions [15]. However, the phenomenon of decay of the quasiparticules is not described by the Landau form (1). Since quasiparticules are true eigenmodes of (1), they have an infinite life-time. By contrast, the form (2) takes into account the decay of the quasiparticules and it is indeed possible to calculate the decay rate of the quasiparticules from the Hamiltonian (2) [16]. Even though the Hamiltonian (2) it is not diagonal, it can be brought to diagonal form using some asumptions and by bosonizing the Fermi surface [18]. We shall review the main assumptions and the bosonization of (2) in the thermodynamic limit and in the limit of a zero curvature of the Fermi surface. One of the ingredients of the solution via bosonization is the existence of a momentum $\Lambda$ which determines the single particule states which participate in the formation of bosons in the vicinity of the Fermi surface. $\Lambda$ is a necessary ingredient, because of the curvature of the Fermi surface, but is also a source of difficulty since the objects with a true bosonic character in the limit $\Lambda \rightarrow+\infty$ are no more exactly bosonic in the limit of a finite momentum cutoff $\Lambda$.

Of course, numerical computations cannot be performed in the thermodynamic limit since one can only diagonalize matrices of size 2000 by a Jacobi method. This technical limitation imposes to work with small systems and to impose a drastic cutoff $\Lambda$, so that the Hamiltonian 
(2) is no more integrable in the framework of the Hilbert space of the numerical diagonalisations. In the case of the presence of a cutoff, and for finite size systems, one may thus expect the universal Poisson level spacing statistics to be replaced by the universal G.O.E. level spacing statistics of the form

$$
P(s)=\frac{\pi}{2} s \exp \left(-\frac{\pi s^{2}}{4}\right) .
$$

It is necessary to determine the importance of the cutoff $\Lambda$ on the level spacing statistics and whether the statistics evolve towards G.O.E. level spacing statistics as $\Lambda$ is reduced. This has not only a numerical interest, but also a physical one. For instance, lattice models have a natural cutoff $\Lambda \sim 1 / a$, where $a$ is the lattice spacing. To answer this question, we come back to one dimensional spinless fermionic systems, but with a cutoff $\Lambda$ and study the level spacing statistics for $g_{2}$ and $g_{4}$ interactions. As we shall see, the level spacing statistics are drastically affected by the presence of the cutoff since the bosonic superpositions of particule-hole pairs which guarantee the integrability, do not survive in the presence of the cutoff. If the cutoff $\Lambda$ is fixed and the size $L$ of the system increases, one expects for a crossover from a G.O.E. level spacing statistics in the high energy part of the spectrum to a Poisson level spacing statistics at low energy, which shows that finite size effects are drastic in the presence of the cutoff. In order to determine the crossover scale between the Poisson regime and the G.O.E. regime, we introduce temperature-dependent level spacing statistics. The statistics at finite temperatures are found to pass from a non integrable statistics at high temperatures to Poisson statistics at low temperature.

As far as two dimensionnal systems are concerned, we carry out numerical diagonalisations of a small system of electrons, with a small number of orbitals. In these conditions, the Hamiltonian (2) is non integrable. We found Poisson level spacing statistics even for a small system. We attribute this property to the fact that the interactions generate extra diagonal matrix elements in the Hamiltonian which are in competition with diagonal interaction matrix elements, but the interaction connects only a small number of states, leaving a lot of zero matrix elements in the Hamiltonian. Provided the off-diagonal matrix elements are of the same order than the diagonal ones, the statistics are dominated by the diagonal matrix elements. However, by keeping only off-diagonal interaction matrix elements, we were able to exhibit G.O.E. level spacing statistics.

This paper is organized as follows. We first treat the one dimensional case, in the Hilbert space restricted by the momentum cut-off $\Lambda$. The results established in one dimension are helpful in two dimensions since the presence of the momentum cut-off is also a source of non integrability. In a second step, we come to the bidimensional Fermi liquids, with the study of the level spacing statistics of the Hamiltonian (2). 


\section{Level spacing statistics of 1 D spinless fermion sys- tems in the $\Lambda$-restricted Hilbert space}

\subsection{Bosonization and level spacing statistics in the unrestricted Hilbert space (Luttinger liquid)}

Using the bosonization procedure of Haldane [22], we can solve the one dimensional spinless Luttinger liquid with a linear dispersion relation and $g_{2}$ and $g_{4}$ interactions. The Hamiltonian reads, in term of fermions

$$
H=v_{F} \sum_{k, \alpha}\left(\alpha k-k_{F}\right): c_{k, \alpha}^{+} c_{k, \alpha}:+\frac{\pi}{L} \sum_{\alpha, \alpha^{\prime}} \sum q\left(g_{4 q} \delta_{\alpha \alpha^{\prime}}+g_{2 q} \delta_{\alpha,-\alpha^{\prime}}\right) \rho_{q, \alpha} \rho_{-q, \alpha^{\prime}},
$$

where the label $\alpha$ indexes the branch $\alpha=\mathrm{R}($ ight $), \mathrm{L}(\mathrm{eft})=+1,-1$. The interaction $g_{4}$ describes the diffusion of two fermions on the same right or left branch whereas $g_{2}$ describes the diffusion of two fermions belonging to the right and left branch. The density operators are defined as

$$
\rho_{q, \alpha}=\sum_{k}: c_{k+q, \alpha}^{+} c_{k, \alpha}:
$$

and obey bosonic commutation relations

$$
\left[\rho_{q, \alpha}, \rho_{q^{\prime}, \alpha^{\prime}}^{+}\right]=-\delta_{\alpha, \alpha^{\prime}} \delta_{q, q^{\prime}} \frac{L \alpha q}{2 \pi},
$$

which allows the definition of boson operators

$$
a_{q}^{+}=\left(\frac{2 \pi}{L|q|}\right)^{1 / 2} \sum_{\alpha} \theta(\alpha q) \rho_{q, \alpha}
$$

and make it possible to diagonalize the Hamiltonian (6) via a Bogoliubov transformation:

$$
H=E_{0}+\sum_{q} \omega_{q} b_{q}^{+} b_{q}+\frac{\pi}{2 L}\left(v_{N} N^{2}+v_{J} J^{2}\right)
$$

where

$$
\begin{aligned}
b_{q}^{+} & =\cosh \varphi_{q} a_{q}^{+}-\sinh \varphi_{q} a_{-q} \\
\tanh 2 \varphi_{q} & =-\frac{g_{2 q}}{v_{F}+g_{4 q}} \\
\omega_{q} & =\left|\left(v_{f}+g_{4 q}\right)^{2}-\left(g_{2 q}\right)^{2}\right|^{1 / 2}|q| \\
v_{S} & =\left(\left(v_{F}+g_{40}\right)^{2}-\left(g_{20}\right)^{2}\right)^{1 / 2} \\
v_{N} & =v_{S} \exp -2 \varphi_{0} \\
v_{J} & =v_{S} \exp 2 \varphi_{0} .
\end{aligned}
$$


The interaction functions $g_{2}(q)$ and $g_{4}(q)$ are supposed to tend to a constant in the limit $q \rightarrow 0$, and to zero in the limit $q \rightarrow+\infty$. Their decrease is controlled by the impulsion scale $2 \pi / R$, where $R$ is a given length scale. In the thermodynamic limit $(L \gg R)$, one can define an effective low energy Hilbert space. To do so, we assume that $\omega_{q} \simeq \omega_{0}$ for all the wave vectors $q$. Of course, this approximation is only valid if the temperature is low enough. In this limit, the Hamiltonian (10) depends only on two velocities $v_{N}$ and $v_{J}$ :

$$
H=E_{0}+\sum_{q \neq 0}\left(v_{N} v_{J}\right)^{1 / 2}|q| b_{q}^{+} b_{q}+\frac{\pi}{2 L}\left(v_{N} N^{2}+v_{J} J^{2}\right) .
$$

In one dimension, the link between the breakdown of the Fermi liquid picture and the level spacing spacing statistics is well understood. The absence of a Fermi surface in one dimension, in the presence of long range $g_{2}$ and $g_{4}$ interactions was known to Dzyaloshinskii and Larkin in the 70's [17]. The breakdown of the Fermi liquid picture is already present at the level of the static correlation functions, and in the Hilbert space of low energy. The usual infrared divergencies are governed by the $q \rightarrow 0$ limit of $g_{2}(q)$ and $g_{4}(q)$, and are related to the orthogonality catastrophy and the absence of a Fermi surface. The infrared spectrum is described by the Hamiltonian (17). If the total charge and current quantum numbers are given, the $q \neq 0$ excitations are bosons with a linear dispersion relation, but with a renormalized velocity. The departure from the gas behaviour is measured by the anomalous exponents which appear in the static Green's functions. The interaction energy scale associated to this static breakdown of the Fermi liquid is given by [13]

$$
g_{2}^{\text {stat }} \sim v_{F}\left(\ln \frac{L}{2 \pi R}\right)^{-1 / 2}
$$

However, as emphasized in 13], the static part of the Green's function does not contain all the physics of the breakdown of the Fermi liquid theory. From a calculation of the two points Green's function, and from a calculation of the switching-on pocedure, we found in [13] a dynamical breakdown of the Fermi liquid picture, controlled by the interaction scale

$$
g_{2}^{d y n} \sim \frac{4 \pi v_{F}}{\left(k L(k R)^{\alpha}\right)^{1 / 2}}
$$

where $\alpha$ is defined by

$$
g_{2 q}=g_{20}\left(1-(q R)^{\alpha}\right) .
$$

The static process of breakdown of the Fermi liquid picture does not modifie the level spacing statistics, which remain singular, as in the case of the gas, since the dispersion relation of the bosons remain linear. By contrast, the dynamical breakdown of the Fermi liquid appears to be related to a cross-over in the energy level spacing statistics, namely from singular level spacing 
statistics to generical Poisson level spacing statistics. As far as symetries are concerned, the gas case and the Luttinger liquid possess the conformal symetry. The static breakdown of the Fermi liquid preserves the conformal invariance. The theory is the antiperiodic-antiperiodic sector of the compactified boson, with an interaction-dependent radius of compactification. The dynamical breakdown of the Luttinger liquid corresponds to a massless loss of conformal invariance [12].

\section{$2.2 \quad$ Fourier transform of the gas spectrum and temperature-dependent level spacing statistics}

We first wish to characterize the spectrum of the gas in the absence of interactions, a momentum cutoff $\Lambda$ and a finite size $L$. The idea is to find a criterium to detect when a boson of given wave vector $q$ is present or not in the system. Since $g_{2}$ and $g_{4}$ interactions are diagonal on the basis of bosonic excitations, this tool is a good way to characterize the degree of integrability of the model with a momentum cut-off. We consider a one branch model, and the number of quantum states is simply the integer part of $2 \Lambda L / 2 \pi$. The spectrum is made up of levels with an equidistant separation $2 \pi v_{F} / L$. In order to characterize the spectrum, we use its Fourier transform

$$
f_{L}(\tau)=\int_{-\infty}^{+\infty} e^{i \omega \tau} \sum_{\left\{E_{i}\right\}} \delta\left(\omega-E_{i}\right)=\sum_{\left\{E_{i}\right\}} e^{i E_{i} \tau} .
$$

In the thermodynamic limit,

$$
f_{\infty}(\tau)=\int_{-\infty}^{+\infty} e^{i \omega \tau} \sum_{\left\{n_{q}\right\}} \delta\left(\omega-v_{f} \sum_{n_{q}=0}^{+\infty} q n_{q}\right),
$$

leading to

$$
\left|f_{\infty}(\tau)\right|=\prod_{q>0} \frac{1}{2\left|\sin v_{f} \tau q / 2\right|} .
$$

This function presents poles which are characteristic of the bosonic modes for $\tau_{n, q}=2 \pi n / v_{f} q$, with $n$ an integer. If we rescale $\tau$ by a factor $2 \pi$ and choose $v_{f}=1$, we obtain a pole for $\tau_{n, q}=n / q$, that is for every rational number. We plotted $\left|f_{\infty}(\tau)\right|$ for 5 bosonic modes on figure 1. One can easily recognize the different bosons in the sequence of poles. In the presence of interactions, the poles are deplaced. We also studied the Fourier transform of the spectrum in the case of a finite size system. The result is depicted on figure 2. We can see that the poles are not so well defined. However, we can recognize the formation of peaks which replace the poles, and attribute well defined boson wave vectors to some peaks. The corresponding truncated density operators are defined by

$$
\rho_{\Lambda}(q)=\sum_{k} \theta_{\Lambda}(k) \theta_{\Lambda}(k+q) c_{k+q}^{+} c_{k}
$$


where $\theta_{\Lambda}(k)=\theta\left(\Lambda-\left|k-k_{f}\right|\right)$. We can ask under which condition a peak corresponding to a truncated boson $\rho_{\Lambda}(q)$ appears on the modulus of the Fourier transform of the spectrum $\left|f_{L}(\tau)\right|$. To answer this question, we assume that the number $n_{q}$ of bosonic excitations of wave vector $q$ appearing in the presence of a cutoff is such as $q n_{q} \sim 2 \Lambda$, so that $f_{L}\left(\tau_{1, q}\right) \sim 2 \Lambda / q$ for the peak at $\tau=\tau_{1, q}$. A boson of wave vector $q$ is well defined provided $f_{L}\left(\tau_{1, q}\right) \gg 1$, that is if $\Lambda \gg q / 2$. For a given cut-off, the statistics are expected to be Poisson at low energies, and G.O.E. at higher energies. This is due to the fact that truncated bosons are created essentially at low energy. In order to test this idea, we introduce temperature-dependent level spacing statistics, which are defined as follows. If $\left\{E_{i}\right\}$ is the full spectrum and $\left\{\epsilon_{i}\right\}$ are the energy levels after the smoothing procedure [23], the density of level spacing $P(s)$ is

$$
P_{\beta}(s)=\left(\sum_{i} \exp \left(-\beta \frac{E_{i+1}+E_{i}}{2}\right)\right)^{-1} \sum_{i} \exp \left(-\beta \frac{E_{i+1}+E_{i}}{2}\right) \delta\left(s-\left(\epsilon_{i+1}-\epsilon_{i}\right)\right) .
$$

Moreover, for the statistics to be comparable, one needs to scale $P_{\beta}(s)$ and $s$ such as $\left\langle P_{\beta}(s)\right\rangle=$ 1 , and $\left\langle s P_{\beta}(s)\right\rangle=1$. The reason why we have to rescale these quantities is that they were equal to unity after the smoothing procedure, which was carried out in the zero temperature limit. This property is no more valid with the statistical weights of equation (25). For an infinite temperature $(\beta=0)$, we recover the usual level spacing statistics. As the inverse temperature $\beta$ increases, the low energy levels carry more and more statistical weight. As we saw from the description in terms of the truncated bosons (24), the spectrum is expected to be integrable at low energies, so that the level spacing statistics should evolve from G.O.E. statistics, or at least intermediate statistics (that is, with $0<P(0)<1$ ), to Poisson level spacing statistics as $\beta$ increases.

\subsection{Level spacing statistics with $g_{2}$ interactions in the $\Lambda$-restricted Hilbert space}

The numerical method to compute the level spacing statistics of two branch models with a momentum cutoff $\Lambda$ and $g_{2}$ interactions consists in tabulating the states of the Hilbert space by generating all the different fillings of the two branches, the number of particules on each branch being kept constant. The second step consists in computing all the matrix elements of the hamiltonian and diagonalising the Hamiltonian by the use of the Jacobi method. The interactions are of the form

$$
g_{2 q}=g_{20} \exp -\left(\frac{q L}{2 \pi R}\right)
$$

The scale of the interactions $R$ is chosen equal to $\Lambda$, so that all the matrix elements of the interaction are important. The evolution of the spectrum as a function of $g_{20}$ is plotted on figure 3. This figure is to be compared with the figure 2 of reference [13], where we have plotted 
the evolution of the energy levels as a function of $g_{20}$ but in the absence of cutoff. The obvious difference between the two plots is the presence of level repulsion with a cutoff $\Lambda$, and the existence of level crossings in the absence of cutoff. The level spacing statistics corresponding to a spectrum analog to the one of figure 3 are plotted on figure 4 . They are in good agreement with G.O.E. level spacing statistics. In our computation, $\Lambda L / 2 \pi=3$, so that the criterium $\Lambda \gg 2 \pi / L$ is not verified. We could not go to higher values of $\Lambda L / 2 \pi$ because increasing the ratio $\Lambda L / 2 \pi$ increases the size of the Hilbert space and numerical diagonalizations are no more possible. We conclude that the statistics depend drastically on the length of the system, if the momentum cutoff is fixed. If we apply the ideas of temperature-dependent statistics of level spacings to the system, we find that the statistics of level spacings are driven from a G.O.E. law at $\beta=0$ to a Poisson law as $\beta$ increases, as depicted on figure 5. This evolution reveals the fact that "nearly integrable" degrees of freedom exist at low energy. These bosonic degrees of freedom are exactly integrable in the absence of cut-off. In term of classical trajectories (provided one is able to find a classical phase space for the Fermi liquid !), this situation corresponds to the existence of conserved torii at low energy, which transform into chaotic trajectories as the energy increases. The cross-over temperature scale shall be derived later.

\subsection{Level spacing statistics with $g_{4}$ interactions in the $\Lambda$-restricted Hilbert space}

We now consider the case of $g_{4}$ interactions. The interaction Hamiltonian is

$$
H^{1}=\sum_{q \neq 0} \sum_{k, k^{\prime} \neq k+q} g_{4 q} c_{k+q}^{+} c_{k^{\prime}-q}^{+} c_{k^{\prime}} c_{k}
$$

The interest of the $g_{4}$ term is that we can use only a one branch model, and we can reach higher values of the ratio $\Lambda L / 2 \pi$ without increasing the size of the Hilbert space. We could reach $\Lambda L / 2 \pi=12$ in a sector of total momentum $P=24.2 \pi / L . \quad P$ is the impulsion with respect to the fundamental. As long as $P \leq \Lambda L / \pi$, one has generated the complete Hilbert space in the absence of interactions. This fact motivates the choice $P=\Lambda L / \pi$. The condition $\Lambda L / \pi=24 \gg 1$ is respected. However, we did not find Poisson statistics, but statistics which are intermediate between a Poisson law and G.O.E. statistics. Namely, the value of the density of normalized zero crossings is not 1 but 0.5 . The statistics are plotted on figure 6. This computation gives an idea of the extension of the crossover as a function of $\Lambda L / 2 \pi$, since we obtain intermediate statistics for $\Lambda L / 2 \pi=24$. We computed also the temperaturedependent level spacing statistics. We found a cross-over to Poisson statistics as the inverse temperature increases, as plotted on figure 0 . This cross-over is much more rapid than in the case of the $g_{2}$ interaction. However, the values of the cutoff in the $g_{2}$ case and the $g_{4}$ case are not comparable. 


\subsection{Cross-over scales}

In order to get an idea of the temperature cross-over between the G.O.E. and Poisson regime, we look for the energy scale $k_{B} T^{*}$ below which the energy levels are decorrelated. To do so, we make the approximation that the only effect of interactions is to compress the spectrum by an amount $\left.\left(\left(1+g_{4} / v_{f}\right)^{2}-\left(g_{2} / v_{f}\right)^{2}\right)\right)^{1 / 2}$, where $g_{2}$ and $g_{4}$ are typical energy interactions. This compression of the spectrum means that the effective mass increases if $g_{2}$ is large compared to $g_{4}$. This approximation corresponds to taking local interactions in the real space. Then, we see that the integrable modes under request are such as

$$
v_{f}|q| \leq\left(\left(1+\frac{g_{4}}{v_{f}}\right)^{2}-\left(\frac{g_{2}}{v_{f}}\right)^{2}\right)^{-1 / 2} k_{B} T .
$$

These modes are integrable provided they lead to well-defined bosons. We say that a boson is well-defined provided a sufficient number of particule-hole excitations enter into the summation (24), that is if $2 \Lambda-|q| \geq 2 \pi \alpha / L$, where $\alpha$ is a dimensionalless coefficient, which counts the number of non-zero terms in (24), and is thus a measure of integrability. We get that

$$
k_{B} T \simeq(2 \Lambda-2 \pi \alpha / L)\left(\left(1+g_{4} / v_{f}\right)^{2}-\left(g_{2} / v_{f}\right)^{2}\right)^{1 / 2}
$$

The energy scale $k_{B} T^{*}$ thus increases as the cut-off $\Lambda$ increases, and decreases if the interaction strength $g_{2}$ increases.

\section{Level spacing statistics of a 2 D spinless fermion sys- tem in the $\Lambda$-restricted Hilbert space}

\subsection{Bosonization of the bidimensional Fermi surface}

The bosonization of the Fermi liquid [19], [20], 221] involves a covering of the Fermi surface by spheres of radius $\Lambda$. Each small sphere is labelled by an integer $\alpha$ and one assumes that the Fermi surface is flat at the scale $\Lambda$. Assuming that $\Lambda \gg 2 \pi / L$, the commutation relations of the operators

$$
\rho_{q, \alpha}=\sum_{\mathbf{q}} \theta_{\alpha}(\mathbf{k}+\mathbf{q} / 2) \theta_{\alpha}(\mathbf{k}-\mathbf{q} / 2) n_{\mathbf{q}}(\mathbf{k})
$$

with

$$
n_{\mathbf{q}}(\mathbf{k})=c_{\mathbf{k}-\mathbf{q} / 2}^{+} c_{\mathbf{k}+\mathbf{q} / 2}
$$

must include a Schwinger term, leading to the commutation relation

$$
\left[\rho_{\mathbf{q}, \alpha}, \rho_{\mathbf{q}^{\prime}, \alpha^{\prime}}^{+}\right]=\delta_{\alpha, \alpha^{\prime}} \delta_{\mathbf{q}, \mathbf{q}^{\prime}} \sum_{k} C_{\alpha}(\mathbf{k}, \mathbf{q})\left(n_{\mathbf{k}+\frac{\mathbf{q}}{2}}^{0}-n_{\mathbf{k}-\frac{\mathbf{q}}{2}}^{0}\right),
$$


where the constraint $C_{\alpha}$ is

$$
C_{\alpha}(\mathbf{k}, \mathbf{q})=\theta_{\alpha}\left(\mathbf{k}+\frac{\mathbf{q}}{2}\right) \theta_{\alpha}\left(\mathbf{k}-\frac{\mathbf{q}}{2}\right) \theta_{\alpha}\left(\mathbf{k}^{\prime}+\frac{\mathbf{q}^{\prime}}{2}\right) \theta_{\alpha}\left(\mathbf{k}^{\prime}-\frac{\mathbf{q}^{\prime}}{2}\right) .
$$

To simplify the expression (32), one makes the assumption of a flat Fermi surface in each sphere of radius $\Lambda$. In order to specify this condition, one imposes that no bosonic excitations with an angle $\theta>\pi / 2$ exist, where $\theta$ denotes the angle between $\mathbf{k}_{\mathbf{f}}$ and $\mathbf{q}$. The maximal angle theta is such as

$$
\tan \left(\theta-\frac{\pi}{2}\right) \leq \frac{2 \pi}{L \Lambda}
$$

which leads to

$$
\Lambda \leq\left(\frac{4 \pi k_{f}}{L}\right)^{1 / 2}
$$

The equation (35) means that the curvature is negligeable in a sphere of radius $\Lambda$, and is compatible with the condition

$$
\Lambda \gg 2 \pi / L
$$

provided the linear length is large enough: $L \gg \pi / k_{f}$. If $\Lambda \ll 2 \pi / L$ and $|\mathbf{q}| \ll \Lambda$, the leading term in (32) is of the form $\left[\rho_{\mathbf{q}, \alpha}, \rho_{\mathbf{q}^{\prime}, \alpha^{\prime}}^{+}\right]=\delta_{\alpha, \alpha^{\prime}} \delta_{\mathbf{q}, \mathbf{q}^{\prime}} V a\left(\mathbf{q} \cdot \mathbf{n}_{\alpha}\right)$. We evaluate the corrections under the conditions $\Lambda \gg 2 \pi / L$ and $|\mathbf{q}| \ll \Lambda$. Under these assumptions, the leading term represents the number of states in the parallelogram of figure 8. To obtain the corrections, one has to substract the number of states contained in the small shaded triangle. The number of states to be removed is approximately equal to

$$
\frac{1}{2}\left(\frac{L}{2 \pi}\right)^{2}|\mathbf{q}|^{2} \cos \theta \sin \theta=V a\left(\mathbf{q} \cdot \mathbf{n}_{\alpha}\right) \cdot \frac{\left|\mathbf{q} \wedge \mathbf{n}_{\alpha}\right|}{2 k_{f}} .
$$

The commutation relations are thus of the form

$$
\left[\rho_{\mathbf{q}, \alpha}, \rho_{\mathbf{q}^{\prime}, \alpha^{\prime}}^{+}\right]=\delta_{\alpha, \alpha^{\prime}} \delta_{\mathbf{q}, \mathbf{q}^{\prime}} V a\left(\mathbf{q} \cdot \mathbf{n}_{\alpha}\right)\left\{1+O\left(\frac{\left|\mathbf{q} \wedge \mathbf{n}_{\alpha}\right|}{k_{f}}\right)\right\} .
$$

Following reference [21], we define

$$
a_{\mathbf{q}}\left(\mathbf{k}_{\mathbf{f}}\right)=\sum_{\mathbf{k}} \phi_{\Lambda}\left(\left|\mathbf{k}-\mathbf{k}_{\mathbf{f}}\right|\right)\left[n_{\mathbf{q}}(\mathbf{k}) \theta\left(\mathbf{q} \cdot \mathbf{v}_{\mathbf{k}}\right)+n_{-\mathbf{q}}(\mathbf{k}) \theta\left(-\mathbf{q} \cdot \mathbf{v}_{\mathbf{k}}\right)\right]
$$

and

$$
b_{\mathbf{q}}\left(\mathbf{k}_{\mathbf{f}}\right)=\left(N_{\Lambda} V\left|\mathbf{q} \cdot \mathbf{v}_{\mathbf{k}}\right|\right)^{-1 / 2} a_{\mathbf{q}}\left(\mathbf{k}_{\mathbf{f}}\right),
$$

where $\phi_{\Lambda}$ is a smearing function such as $\phi_{\Lambda} \rightarrow \delta_{\mathbf{k}, \mathbf{k}_{\mathbf{f}}}$ if $\Lambda \rightarrow 0$, and $N_{\Lambda}$ is the local density of states:

$$
N_{\Lambda}=\frac{1}{V} \sum_{\mathbf{k}} \mid \phi_{\Lambda}\left(\left|\mathbf{k}-\mathbf{k}_{\mathbf{f}}\right|\right)^{2} \delta\left(\mu-\epsilon_{\mathbf{k}}\right)
$$


Provided the curvature of the Fermi surface is negligeable, that is provided condition (35) is satisfied, one can bosonize the Hamiltonian (2) to obtain

$$
H=\sum_{\mathbf{k}_{\mathbf{f}}} \sum_{\mathbf{q}, \mathbf{q} \cdot \mathbf{v}_{\mathbf{k}}>0}\left|\mathbf{q} \cdot \mathbf{v}_{\mathbf{k}}\right| b_{\mathbf{q}}^{+}\left(\mathbf{k}_{\mathbf{f}}\right) b_{\mathbf{q}}\left(\mathbf{k}_{\mathbf{f}}\right)+\frac{1}{2 V} \sum_{\mathbf{k}, \mathbf{k}^{\prime}, \mathbf{q}} f_{\mathbf{k}, \mathbf{k}^{\prime}, \mathbf{q}} n_{-\mathbf{q}}(\mathbf{k}) n_{\mathbf{q}}\left(\mathbf{k}^{\prime}\right)
$$

Since the interactions are quadratic in the boson operators, one can diagonalize them via a generalized Bogoliubov transformation. However, in numerical computations, one can only treat a Hilbert space of size 2000. This means that the cutoff $\Lambda$ must be reduced drastically, as well as the Fermi wave vector $k_{f}$. In particular, the conditions (35) and (36) are no longer valid and one cannot diagonalize the Hamiltonian as described above. Two scenarios are candidates for the appearance of chaos in the spectrum of the bidimensional Fermi liquid. The first scenario is the effect of curvature. In the limit $L \rightarrow+\infty$, and for a cut-off independent of $L$, the condition (35) is no more valid, and one cannot solve the model by bosonization. We shall not study this effect in the present paper. The second scenario comes from the fact that, even with a flat Fermi surface, the condition (36) may be violated, so that the system is no more integrable by bosonization. We shall study the last type of effect in the rest of the paper.

\subsection{Level spacing statistics of a $2 \mathrm{D}$ spinless fermion system in the $\Lambda$-restricted Hilbert space}

We now turn to the bidimensional case in the presence of a momentum cutoff. The Fermi sea in its fundamental state is pictured on the insert of figure 9. We treated a Fermi sea of 5 electrons for a total of 29 available quantum states. The Hamiltonian is given by the expression (2). The interaction term can be split into two terms as follows

$$
\begin{aligned}
H^{1} & =H_{0}^{1}+H_{1}^{1} \\
H_{0}^{1} & =-\frac{1}{V} \sum_{\mathbf{k}, \mathbf{q}} f_{\mathbf{k}, \mathbf{k}+\mathbf{q}, \mathbf{q}} n_{\mathbf{k}+\mathbf{q}} n_{\mathbf{k}} \\
H_{1}^{1} & =\frac{1}{V} \sum_{q \neq 0} \sum_{\mathbf{k}, \mathbf{k}^{\prime} \neq \mathbf{k}+\mathbf{q}} f_{\mathbf{k}, \mathbf{k}^{\prime}, \mathbf{q}} c_{\mathbf{k}+\mathbf{q}}^{+} c_{\mathbf{k}^{\prime}-\mathbf{q}}^{+} c_{\mathbf{k}^{\prime}} c_{\mathbf{k}}
\end{aligned}
$$

The term $H_{0}^{1}$ is of the same nature as the diagonal Landau interaction between quasiparticules. The only difference is that $\delta n_{k}$ represents occupation numbers of renormalized quasiparticules in the Landau theory, whereas $n_{k}$ is the number operator of bare fermions. The Hamiltonian made up of the kinetic term $H^{0}$ (3) plus the term $H_{0}^{1}$ (44) has already been studied in reference [11] and leads to Poisson statistics in two dimensions. In the limit in which the bidimensional 
bosonization procedure is applicable, it has been established in [21] that the Hamiltonian $H+H_{0}^{1}+H_{1}^{1}$ is integrable, namely that the effect of $H_{0}^{1}+H_{1}^{1}$ is to renormalize the free theory without breaking the integrability. We question whether this property is still valid for a system of electrons such as the one drawn on the insert of figure 9. To see this, we diagonalize the Hamiltonian $H^{0}+H_{0}^{1}+H_{1}^{1}$ for the electron system of the insert of figure 9, in a sector of

fixed total momentum. As expected from bosonization [21] and from R.P.A. theory [24, we obtain a collective bound state which detaches itself from the particule-hole continuum. The energy of the collective mode is greater as the continuum energy for repulsive interactions, and lower for attractive interactions. Because of the small value of the momentum cutoff $\Lambda$, the energy width of the continuum is small compared to the energy of the bound state, so that the bound state renormalizes the level spacing statistics of the continuum towards small values, since the mean value of the level spacing distributions is rescaled to unity. If we suppress the bound state from the spectrum, we obtain level spacing statistics in good agreement with Poisson level spacing statistics, as shown on figure 9. It shows that whereas the cutoff $\Lambda$ is very small and the number of electrons small too, the result we obtain is consistent with the result predicted in the limit in which the bosonization procedure is valid, with a large cutoff compared to $2 \pi / L$. However, there is no contradiction with what has been done in one dimension. If we assume that in two dimensions the statistics are poissonian provided a sufficient number of truncated bosons (30) have a sufficient number of non zero terms in their linear combination, we obtain the same criterium as in one dimension, namely that the statistics are poissonian provided $\Lambda \gg 2 \pi / L$. Using the cross-over scale we obtained from the diagonalization of $g_{4}$ interactions, we reach the conclusion that the level spacing statistics of the Hamiltonian $H^{0}+H_{2}^{1}$ should be of the G.O.E. type. This is indeed the case, as shown on figure 10. We attribute the fact that the level spacing statistics of $H^{0}+H_{0}^{1}+H_{1}^{1}$ are poissonian to the presence of only a small number of off-diagonal terms, which are not numerous enough to change significatively the statistics.

\section{Conclusion}

In this paper, we have studied the effect of the momentum cutoff on the level spacing statistics of interacting Fermi systems. We found that the presence of the cutoff could change drastically the level spacing statistics of a finite size system, namely to drive the level spacing statistics from a poissonian shape to G.O.E. statistics. Using temperature-dependant level spacing statistics and one dimensional models, we have shown that the system of electrons in the presence of a cutoff is integrable at low energy. As far as $g_{2}$ interactions are concerned, we obtained G.O.E. level spacing statistics, which evolve to a Poisson level spacing statitics as the temperature decreases from $+\infty$. In the $g_{4}$ case, we could reach higher values of the cutoff 
since the two branches of the dispersion relation are decoupled. The infinite temperature level spacing statistics were intermediate between Poisson and G.O.E., and were driven to a Poisson shape as the temperature decreases. The main feature of a system of spinless correlated fermions in the presence of a momentum cutoff in the $k$-space is that, inspite of the loss of integrability there subsists nearly uncorrelated levels at low energy. This conclusion is analogous to the conclusions of reference [25] for another model. In two dimensions, one has to distinguish between two cases. The first case corresponds to off-diagonal interactions only. In this case, the level spacing statistics are G.O.E. statistics. It is clear that in this case, no switching on procedure can connect the excitations of the gas and the excitations of the interacting system, since the nature of the two spectra is different, which means that the system is not at the Landau fixed point. In the second case, diagonal interactions of the Landau type are taken into account. The Poisson level spacing statistics are restored because the off-diagonal matrix elements are not numerous enough, whereas their amplitude is comparable to the amplitude of the diagonal matrix elements. The Fermi liquid behaviour of the Hamiltonian in the presence of diagonal interactions is thus not destroyed. This result is to be compared with the fact that the Hamiltonian is diagonal in the limit in which the bidimensional bosonization procedure is valid. In spite of the small number of fermions and the small number of orbitals in our numerical computations, we obtain a result in good agreement with the bosonization of the Fermi surface theory. It should be stressed that the limit in which the bosonization theory is valid is not the thermodynamic limit in which $\Lambda$ is fixed and $L \rightarrow+\infty$, because of the constraint (35). The problem of the thermodynamic limit, with the condition (35) violated remains open.

The finite temperature level spacing statistics seem to be an appealing tool for the study of the spectrum of strongly correlated electronic systems. From a finite size study, one can characterize the integrability at low energy, and the cross-over temperature measures how far the low energy degrees of freedom are from being integrable. However, it is clear that the level spacing statistics retain only information about the the symetries, and give no information about the decay of the correlation functions, so that, in principle, we cannot solve entirely the problem whether the bidimensional t-J model is a Fermi liquid or not. Nonetheless, we can characterize the degree of integrability of the low energy degrees of freedom. A forthcoming paper shall be devoted to the case of finite size t-J models [10].

The author acknowledges B. Douço for underlying the role of the momentum cutoff, and J.C. Anglès d'Auriac for help with algorithms. 


\section{References}

[1] G. Montambaux, D. Poilblanc, J. Bellisard and C. Sire, Phys. Rev. Lett. $\underline{70}, 497$ (1993).

[2] T.C. Hsu and J.C. Anglès d'Auriac, Phys. Rev. B $\underline{47}, 14291$ (1993).

[3] D. Poilblanc, T. Ziman, J. Bellissard, F. Mila and G. Montambaux, Europhys. Lett. 22 , 537 (1993).

[4] P.W. Anderson, Phys. Rev. Lett. $\underline{65}$, 2306 (1990).

[5] J.R. Engelbrecht and M. Randeria, Phys. Rev. Lett. $\underline{65}$, 1032 (1990).

[6] P.W. Anderson; J.R. Engelbrecht and M. Randeria, Phys. Rev. Lett. $\underline{66}, 3225$ (1991).

[7] E. Dagotto, A. Nazarenko and M. Boninsegni, Phys. Rev. Lett. $\underline{73}, 728$ (1994).

[8] A. Morea, S. Haas, A. Sandwik and E. Dagotto, preprint (1994).

[9] Sorella, preprint cond-mat/9308001.

[10] R. Mélin, in preparation.

[11] R. Mélin, J. Phys. I France ㅁ, 159(1995).

[12] P. Degiovanni and R. Mélin, unpublished.

[13] R. Mélin, B. Douçot and P. Butaud, J. Phys. I France $\underline{4}, 737$ (1994).

[14] Anderson, Basic Notions of Condensed Matter Physics, Frontiers in Physics, The Benjamin/Cummings Publishing Company (1984).

[15] D. Pines and P. Nozieres, The Theory of Quantum Liquids, vol 1, Addison-Wesley Publishing Company $(1966,1989)$.

[16] A. Houghton, H.J. Kwon and J. B. Marston, preprint (1993).

[17] I. E. Dzyaloshinskii and A.I. Larkin Sov. Phys. JETP $\underline{38}, 202$ (1974)

[18] Castro Neto and E. Fradkin, to be published.

[19] F.D.M. Haldane, unpublished.

[20] A. H. Castro Neto and E. H. Fradkin, Phys. Rev. Lett. $\underline{72}, 1393$ (1994).

[21] A. H. Castro Neto and E. H. Fradkin, to be published. 


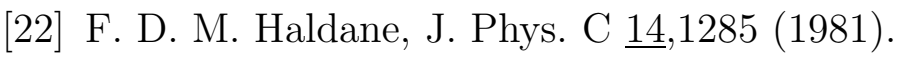

[23] M. C. Gutzwiller, Chaos in Classical and Quantum Mechanics, Springer Verlag (1990).

[24] J.W. Negele and H. Orland, Quantum Many Particule Systems, Frontiers in Physics (Vol. 68), Addison-Wesley Publishing Compagny, Inc (1987).

[25] M. Di Stasio and X. Zotos, to be published. 


\section{Figure captions}

\section{Figure 1:}

Modulus of the Fourier transform of the spectrum in the thermodynamic limit $\left|f_{\infty}(\tau)\right|$ for 5 bosons. Each pole corresponds to one or several definite boson excitations. We have chosen $v_{f}=1$ and $L=2 \pi$. A given boson $q>0$ generates a sequence of poles at $\tau_{n, q}=2 \pi n / q$. The poles are denoted by their corresponding rational number $n / q$.

Figure 2:

Finite size effects of the modulus of the Fourier transform of the spectrum $\left|f_{L}(\tau)\right|$. We have chosen $v_{f}=1$ and $L=2 \pi, \Lambda=11$. As shown on the figure, we can attribute boson quantum numbers to some peaks. We recognize the fractions $1 / 5,1 / 4,1 / 3,2 / 5,1 / 2,3 / 5,2 / 3,3 / 4,4 / 5$.

\section{Figure 3:}

Evolution of the energy levels in the presence of $g_{2}$ interactions with a momentum cutoff. The parameters of the model are $v_{f}=1$ and $L=2 \pi$. The cutoff is chosen equal to 3 , so that we have 3 fermions on each branch, for 6 available quantum states. The interactions are of the form $g_{2 q}=g_{20} \exp (-q / R)$. with $R=6$. The momentum sector is 1 , leading to 45 states in the Hilbert space. Because of the particule-hole symmetry, the spectrum is symmetric, but with no level crossings.

\section{Figure 4:}

Level spacing statistics of $1 \mathrm{D}$ spinless fermions with $g_{2}$ interactions and a cutoff. The parameters are $v_{f}=1, L=2 \pi$. In order to eliminate the particule-hole symmetry present on figure 3, we chose a non symmetric cutoff for particules and holes: the number of fermions on the right branch is 4 , and the number of quantum states is 9 . On the left branch, the number of fermions is 5 for 9 quantum states. The Hilbert space contains 1052 states in the sector of momentum 1. The level spacing statistics are found to be well fitted by G.O.E. statistics. 
Figure 5:

Level spacing statistics of $1 \mathrm{D}$ spinless fermions with $g_{2}$ interactions and a cutoff at finite temperature. The parameters are $v_{f}=1, L=2 \pi$. In order to eliminate the symmetry present on figure 3, we chose a non symmetric cutoff for particules and holes: the number of fermions on the right branch is 4 , and the number of quantum states is 9 . On the left branch, the number of fermions is 5 for 9 quantum states. The Hilbert space contains 1052 states in the sector of momentum 1 . The level spacing statistics are plotted for inverse temperature equal to $\beta=0,0.1,0.5 \mathrm{~A}$ cross-over is found from G.O.E. statistics as $\beta=0$ to Poisson statistics as $\beta$ increases.

Figure 6:

Level spacing statistics of $1 \mathrm{D}$ spinless fermions with $g_{4}$ interactions and with a momentum cutoff. The parameters are chosen such as $v_{F}=1$ and $L=2 \pi$. The value of the momentum cutoff $\Lambda$ is 12 and the total momentum is 24 . The Hilbert space contains 1185 states. The statistics is found to be intermediate between a G.O.E. statistics and a Poisson statistics.

Figure 7:

Level spacing statistics of $1 \mathrm{D}$ spinless fermions with $g_{4}$ interactions and with a momentum cutoff. The parameters are chosen such as $v_{F}=1$ and $L=2 \pi$. The value of the momentum cutoff $\Lambda$ is 12 and the total momentum is 24 . The Hilbert space contains 1185 states. The statistics are plotted for the inverse temperature equal to $\beta=0$ and $\beta=0.3$. The statistics for $\beta=0.3$ is close to the Poisson statistics.

Figure 8:

Representation of a sphere at the Fermi surface. The commutation relation (32) is proportional to the number of states contained in the intersection of the parallelogram and the sphere $n$. The leading order term takes into account all the states in the parallelogram.

Figure 9:

Level spacing statistics of the Hamiltonian $H^{0}+H_{0}^{1}+H_{1}^{1}$. The level spacing statistics are poissonian if one excludes the bound state, and renormalized towards $s=0$ in the presence of the bound state. The linear size of the box is $2 \pi$. The kinetic term $\epsilon(k)$ is quadratic, with a mass equal to 3 . The interactions have the form $f_{k, k^{\prime}, q}=f^{0} \exp (-|q| / R)$, with $R=6$. The insert represents the Fermi sea which was used. The Hilbert space contains 1042 states in the sector of momentum $P=(2 \pi / L)(2,1)$.

Figure 10:

Level spacing statistics of the Hamiltonian $H^{0}+H_{1}^{1}$ with the bound state excluded. The number of zero level spacing is equal to 1114 , leaving only 186 non zero level spacings for the statistics, which explains the important fluctuations of the level spacing statistics which agree with the G.O.E. shape. The insert represents the Fermi sea which was used. The Hilbert space contains 1042 states in the sector of momentum $P=(2 \pi / L)(2,1)$. 